\title{
SCHATTEN CLASS CONDITIONS FOR FUNCTIONS OF SCHRÖDINGER OPERATORS
}

\author{
RUPERT L. FRANK AND ALEXANDER PUSHNITSKI
}

\begin{abstract}
We consider the difference $f\left(H_{1}\right)-f\left(H_{0}\right)$, where $H_{0}=-\Delta$ and $H_{1}=-\Delta+V$ are the free and the perturbed Schrödinger operators in $L^{2}\left(\mathbb{R}^{d}\right)$, and $V$ is a real-valued short range potential. We give a sharp sufficient condition for this difference to belong to a given Schatten class $\mathbf{S}_{p}$, depending on the rate of decay of the potential and on the smoothness of $f$ (stated in terms of the membership in a Besov class). In particular, for $p>1$ we allow for some unbounded functions $f$.
\end{abstract}

\section{INTRODUCTION AND MAIN RESULTS}

1.1. Overview. Let $H_{0}$ and $H_{1}$ be the free and the perturbed (self-adjoint) Schrödinger operators,

$$
H_{0}=-\Delta, \quad H_{1}=-\Delta+V \quad \text { in } L^{2}\left(\mathbb{R}^{d}\right), d \geq 1,
$$

where the real-valued potential $V$ satisfies the bound

$$
|V(x)| \leq C(1+|x|)^{-\rho}, \quad \rho>1 .
$$

The purpose of this paper is to give sharp sufficient conditions for the boundedness and the Schatten class membership of the difference

$$
D(f):=f\left(H_{1}\right)-f\left(H_{0}\right)
$$

where $f$ is a complex-valued function on $\mathbb{R}$ of an appropriate class. These conditions are given in terms of the smoothness of $f$ and the exponent $\rho$ in (1.2). This paper is a continuation of 4, where this problem was considered in the general operator theoretic context. It is also a further development of [3, where the trace class membership of $D(f)$ was considered; we refer to the latter paper for the discussion of applications to mathematical physics.

As it is well known, the continuous spectrum of both $H_{0}$ and $H_{1}$ consists of the closed positive half-line $[0, \infty)$. We focus on the local behaviour of $f$ on $(0, \infty)$. The questions of the behaviour of $f$ at $+\infty$ and near zero are of a very different nature, so in what follows we assume that $f$ is compactly supported on $(0, \infty)$.

If $f$ is sufficiently smooth, say, $f \in C_{0}^{\infty}(0, \infty)$, and the exponent $\rho$ is sufficiently large, then it is not difficult to show, by a variety of standard methods, that the difference $D(f)$ is trace class. On the other hand, as shown in [10], if $f$ has a

Date: 17 January 2019. 
jump discontinuity at a point $\lambda>0$, then $D(f)$ is never compact, unless scattering at energy $\lambda$ is trivial. Thus, a question arises how the transition from the non-compact to the compact difference $D(f)$ occurs when the smoothness of $f$ increases. The "degree of compactness" of $D(f)$ will be measured by its Schatten class membership, and the "degree of smoothness" of $f$ - by its Besov class membership.

Our key example is of $f$ having an isolated cusp-like singularity (see (1.3), (1.4) below) on the positive half-line, smooth elsewhere and compactly supported.

1.2. Boundedness and compactness of $D(f)$. Below $\mathrm{BMO}(\mathbb{R})$ is the class of functions of bounded mean oscillation on $\mathbb{R}$, and $\operatorname{VMO}(\mathbb{R})$ (vanishing mean oscillation) is the closure of $C(\mathbb{R}) \cap \mathrm{BMO}(\mathbb{R})$ in BMO. Further, $\mathcal{B}$ and $\mathbf{S}_{\infty}$ are the classes of bounded and compact operators on $L^{2}\left(\mathbb{R}^{d}\right)$. Precise definitions are given in Section 2 .

Theorem 1.1. Let $H_{0}, H_{1}$ be as in (1.1), (1.2) with $\rho>1$.

(i) For any $f \in \mathrm{BMO}(\mathbb{R})$ with compact support in $(0, \infty)$, we have $D(f) \in \mathcal{B}$.

(ii) For any $f \in \mathrm{VMO}(\mathbb{R})$ with compact support in $(0, \infty)$, we have $D(f) \in \mathbf{S}_{\infty}$.

To illustrate the type of admissible singularities for the function $f$ in the above theorem, let us consider the following example. Let $\chi_{0} \in C_{0}^{\infty}(\mathbb{R})$ be a function which equals 1 in a neighbourhood of the origin and vanishes outside the interval $(-c, c)$ with some $0<c<1$. Then the function

$$
f(x)=\chi_{0}(x)|\log | x||
$$

is in $\operatorname{BMO}(\mathbb{R})$, and the function

$$
f_{\gamma}(x)=\left.\chi_{0}(x)|\log | x\right|^{\gamma}
$$

is in $\operatorname{VMO}(\mathbb{R})$ if $\gamma<1$. Of course, the same applies to all shifted functions $f(x-\lambda)$, $f_{\gamma}(x-\lambda)$ for $\lambda \in \mathbb{R}$. Observe that these functions are unbounded (for $\gamma>0$ ); this is perhaps the most striking feature of Theorem 1.1. Observe also that functions with a jump discontinuity are in BMO, but not in VMO.

1.3. Schatten class membership of $D(f)$. For $0<p<\infty, B_{p, p}^{1 / p}(\mathbb{R})$ is the Besov class of functions on $\mathbb{R}$ and $\mathbf{S}_{p}$ is the Schatten class of all compact operators in $L^{2}\left(\mathbb{R}^{d}\right)$; see Section 2,

Theorem 1.2. Let $H_{0}, H_{1}$ be as in (1.1), (1.2).

(i) Assume $1<\rho \leq d$. Then for any $p>\frac{d-1}{\rho-1}$ and for any $f \in B_{p, p}^{1 / p}(\mathbb{R})$ with compact support in $(0, \infty)$, we have $D(f) \in \mathbf{S}_{p}$.

(ii) Assume $\rho>d$. Then for any $p>d / \rho$ and for any $f \in B_{p, p}^{1 / p}(\mathbb{R})$ with compact support in $(0, \infty)$, we have $D(f) \in \mathbf{S}_{p}$. 
For $p=1$, this is the main result of [3].

To illustrate the type of local singularities allowed for the functions $f \in B_{p, p}^{1 / p}(\mathbb{R})$, consider the following example. Let $\chi_{0} \in C_{0}^{\infty}(\mathbb{R})$ be as above; fix $\alpha>-1, a_{+}, a_{-} \in$ $\mathbb{C}$, and consider the function

$$
F_{\alpha}(x)= \begin{cases}a_{+} \chi_{0}(x)|\log | x||^{-\alpha}, & x>0, \\ a_{-} \chi_{0}(x)|\log | x||^{-\alpha}, & x<0 .\end{cases}
$$

It can be shown that (see [9] or [4, Proposition 1.3])

(i) If $a_{+} \neq a_{-}$and $\alpha>0$, then $F_{\alpha} \in B_{p, p}^{1 / p}(\mathbb{R})$ if and only if $p>1 / \alpha$.

(ii) If $a_{+}=a_{-} \neq 0$ and $\alpha>-1$, then $F_{\alpha} \in B_{p, p}^{1 / p}(\mathbb{R})$ if and only if $p>1 /(\alpha+1)$. We see that for $p>1$, the functions $F_{\alpha}$ may be unbounded. On the other hand, for $0<p \leq 1$, the functions in $B_{p, p}^{1 / p}(\mathbb{R})$ are always bounded and continuous.

1.4. Discussion and some ideas of the proof. Prior to our work [3], the sharpest sufficient conditions for Schatten class inclusions for $D(f)$ were obtained through general operator theoretic estimates of the form [8]

$$
\left\|f\left(H_{1}\right)-f\left(H_{0}\right)\right\|_{p} \leq C(p)\|f\|_{\operatorname{Lip}(\mathbb{R})}\left\|H_{1}-H_{0}\right\|_{p}, \quad 1<p<\infty,
$$

with appropriate modifications for $p=1$ and $p=\infty$ (see [7]). Here $\operatorname{Lip}(\mathbb{R})$ is the Lipschitz class and $\|\cdot\|_{p}$ is the norm in $\mathbf{S}_{p}$. Of course, for the Schrödinger operator, the difference $V=H_{1}-H_{0}$ is never in $\mathbf{S}_{p}$, but one can apply (1.5) to the resolvents of $H_{0}, H_{1}$ (or their powers).

Observe that none of the functions (1.3), (1.4) is in $\operatorname{Lip}(\mathbb{R})$ (unless $\alpha=0$ ); they are not even in any Hölder class. So one cannot hope to prove Theorem 1.2 from (1.5).

In [3], we have used an ad hoc calculation, combining Kato smoothness with an integral representation for $B_{1,1}^{1}$ functions to prove Theorem 1.2 for $p=1$. In [4] we approach the problem in a more systematic fashion; working in a general operator theoretic framework, we introduce the notion of $\mathbf{S}_{p}$-valued Kato smoothness and combine it with the double operator integral technique of Birman and Solomyak to treat all cases $0<p<\infty$; see Sections 2.4 and 2.5 below. Here we apply and adapt the general results of [4] to the Schrödinger operators $H_{0}, H_{1}$.

We proceed as follows. Let $\Lambda$ be an open bounded interval in $\mathbb{R}$, such that supp $f \subset \Lambda$ and the closure of $\Lambda$ is included in $(0, \infty)$. We denote by $\mathbb{1}_{\Lambda}$ (resp. by $\mathbb{1}_{\Lambda^{c}}$ ) the characteristic function of $\Lambda$ (resp. of the complement $\Lambda^{c}$ ) in $\mathbb{R}$. We write

$$
\begin{aligned}
D(f)=\left(\mathbb{1}_{\Lambda}\left(H_{1}\right)\right. & \left.+\mathbb{1}_{\Lambda^{c}}\left(H_{1}\right)\right) D(f)\left(\mathbb{1}_{\Lambda}\left(H_{0}\right)+\mathbb{1}_{\Lambda^{c}}\left(H_{0}\right)\right) \\
& =\mathbb{1}_{\Lambda}\left(H_{1}\right) D(f) \mathbb{1}_{\Lambda}\left(H_{0}\right)-\mathbb{1}_{\Lambda^{c}}\left(H_{1}\right) f\left(H_{0}\right)+f\left(H_{1}\right) \mathbb{1}_{\Lambda^{c}}\left(H_{0}\right) ;
\end{aligned}
$$

here a couple of terms vanish because of the assumption supp $f \subset \Lambda$. We estimate the "diagonal term" $\mathbb{1}_{\Lambda}\left(H_{1}\right) D(f) \mathbb{1}_{\Lambda}\left(H_{0}\right)$ by directly applying the results of [4] and some variants of the limiting absorption principle. We estimate the "off-diagonal 
terms" (the second and third terms in the right side of (1.6)) by using rather standard Schatten class bounds for Schrödinger operators.

Following the proofs, it is not difficult to obtain estimates for the relevant norms of $D(f)$ in terms of the exponents $p, \rho, d$, and the geometry of the support of $f$. However, these estimates are clearly very far from being optimal (perhaps with the exception of the ones for the diagonal term in (1.6) above), and so we have not attempted to work them out explicitly.

1.5. The structure of the paper. The paper can be divided into two parts: in Sections 2 3, we work in a general operator theoretic framework, and in Sections 4 6] we specialise to the case of the Schrödinger operator.

In Section 2 we recall definitions of relevant function and operator classes, discuss the notions of Kato smoothness and $\mathbf{S}_{p}$-valued Kato smoothness and recall the main results of [4, which apply to estimates for the diagonal terms in (1.6). In Section 3, we prove the estimates for the off-diagonal terms in (1.6).

In Section 4 we give sufficient conditions for $\mathbf{S}_{p}$-valued smoothness in the context of the Schrödinger operator. In Section 5 we prove that certain auxiliary operators belong to relevant $\mathbf{S}_{p}$ classes; these facts are needed to treat the off-diagonal terms in (1.6). Finally, in Section 6 we put everything together and prove Theorems 1.1 and 1.2 ,

Acknowledgements. Partial support by U.S. National Science Foundation DMS-1363432 (R.L.F.) is acknowledged. A.P. is grateful to Caltech for hospitality.

\section{Preliminaries}

2.1. The classes BMO and VMO. The space $\mathrm{BMO}(\mathbb{R})$ (bounded mean oscillation) consists of all locally integrable functions $f$ on $\mathbb{R}$ such that the following supremum over all bounded intervals $I \subset \mathbb{R}$ is finite:

$$
\sup _{I}\left\langle\left|f-\langle f\rangle_{I}\right|\right\rangle_{I}<\infty, \quad\langle f\rangle_{I}=|I|^{-1} \int_{I} f(x) d x .
$$

Observe that this supremum vanishes on constant functions. Strictly speaking, the elements of $\mathrm{BMO}(\mathbb{R})$ should be regarded not as functions but as equivalence classes $\{f+$ const $\}$. However, since here we are interested in compactly supported functions $f$, this issue is not important to us. Functions in $\operatorname{BMO}(\mathbb{R})$ belong to $L^{p}(-R, R)$ for any $R>0$ and any $p<\infty$, but not for $p=\infty$ : they may have logarithmic singularities, see (1.3).

Many explicit equivalent norms on $\mathrm{BMO}(\mathbb{R})$ are known (see e.g. [5]). The easiest one to define is the supremum in (2.1). In [4 we use the norm related to Fefferman's duality theorem, which identifies $\mathrm{BMO}(\mathbb{R})$ with the dual to the Hardy class $H^{1}$. This choice of the norm allowed us to explicitly determine the optimal constant appearing in the right hand side of (2.8) . However, in this paper we do not attempt 
to keep track of all constants appearing in estimates, and so the choice of the norm in $\mathrm{BMO}(\mathbb{R})$ is not important here.

The subspace $\operatorname{VMO}(\mathbb{R}) \subset \mathrm{BMO}(\mathbb{R})$ is characterised by the condition

$$
\limsup _{\epsilon \rightarrow 0}\left\langle|f| \leq \epsilon \in\left|\langle f\rangle_{I}\right|\right\rangle_{I}=0 .
$$

Alternatively, $\operatorname{VMO}(\mathbb{R})$ is the closure of $C(\mathbb{R}) \cap \operatorname{BMO}(\mathbb{R})$ in $\operatorname{BMO}(\mathbb{R})$.

In [4], we also use the space $\mathrm{CMO}(\mathbb{R})$ (continuous mean oscillation) which can be characterised as the closure of $C_{\text {comp }}(\mathbb{R}) \cap \operatorname{BMO}(\mathbb{R})$ in $\operatorname{BMO}(\mathbb{R})$. However, for a compactly supported function $f$, conditions $f \in \mathrm{VMO}$ and $f \in \mathrm{CMO}$ coincide.

2.2. The Besov class $B_{p, p}^{1 / p}$. Let $w \in C_{0}^{\infty}(\mathbb{R}), w \geq 0$, be a function with supp $w \subset$ $[1 / 2,2]$ and such that

$$
\sum_{j \in \mathbb{Z}} w_{j}(x)=1, \quad x>0, \quad \text { where } w_{j}(x)=w\left(x / 2^{j}\right) .
$$

The (homogeneous) Besov class $B_{p, p}^{1 / p}(\mathbb{R})$ is defined as the space of tempered distributions $f$ on $\mathbb{R}$ such that

$$
\|f\|_{B_{p, p}^{1 / p}}^{p}:=\sum_{j \in \mathbb{Z}} 2^{j}\left(\left\|f * \widehat{w}_{j}\right\|_{L^{p}(\mathbb{R})}^{p}+\| f *{\widehat{w_{j}}}_{L^{p}(\mathbb{R})}^{p}\right)<\infty .
$$

Here $\widehat{w}_{j}$ is the Fourier transform of $w_{j}$, and $*$ is the convolution.

We will only be interested in compactly supported elements in $B_{p, p}^{1 / p}(\mathbb{R})$. For compactly supported functions $f$, sufficient conditions for Besov class membership can be given in terms of the usual Sobolev spaces:

$$
f \in W_{p}^{s}(\mathbb{R}) \Rightarrow f \in B_{p, p}^{1 / p}(\mathbb{R}), \quad s>1 / p
$$

(For $p \geq 2$, this follows from [1, Theorem 6.4.4], even with $s=1 / p$. For $0<p<2$, this follows from a slight modification of [1, Lemma 6.2.1(1)].) On the other hand, it may be useful to note that

$$
f \in B_{p, p}^{1 / p}(\mathbb{R}) \Rightarrow f \in W_{p}^{1 / p}(\mathbb{R}), \quad 0<p \leq 1 .
$$

(Again, this follows from an adaptation of [1, Lemma 6.2.1(1)] to $0<p \leq 1$.) In particular, $B_{1,1}^{1}(\mathbb{R}) \subset C(\mathbb{R})$.

2.3. Schatten classes. For $0<p<\infty$, the Schatten class $\mathbf{S}_{p}$ is the class of all compact operators $A$ in a given Hilbert space such that

$$
\|A\|_{p}=\left(\sum_{n=1}^{\infty} s_{n}(A)^{p}\right)^{1 / p}<\infty,
$$

where $\left\{s_{n}(A)\right\}_{n=1}^{\infty}$ is the sequence of all singular values of $A$, enumerated with multiplicities taken into account. The expression $\|\cdot\|_{p}$ is a norm for $p \geq 1$ and a 
quasinorm for $0<p<1$. For $0<p \leq 1$ we have the following modified triangle inequality in $\mathbf{S}_{p}$ :

$$
\|A+B\|_{p}^{p} \leq\|A\|_{p}^{p}+\|B\|_{p}^{p}, \quad A, B \in \mathbf{S}_{p}, \quad 0<p \leq 1 .
$$

We will also need the following Hölder inequality in Schatten classes:

$$
\|A B\|_{p} \leq\|A\|_{q}\|B\|_{r}, \quad \frac{1}{p}=\frac{1}{q}+\frac{1}{r} .
$$

2.4. Kato smoothness. Here we briefly recall (with minor simplifications) the relevant definitions and main results of [4].

To motivate what comes next, we should explain that we will factorise the potential $V$ in the form

$$
V=(\operatorname{sign} V)|V|^{1-\theta}|V|^{\theta}
$$

with an appropriate exponent $\theta \in(0,1)$. This corresponds to the "abstract" factorisation

$$
V=G_{1}^{*} G_{0}
$$

of [4]. In [4], we consider the general case, where $G_{0}, G_{1}$ are possibly unbounded operators from a Hilbert space $\mathcal{H}$ to another Hilbert space $\mathcal{K}$, such that $G_{0}$ is $H_{0^{-}}$ bounded and $G_{1}$ is $H_{1}$-bounded. In this paper, since $V$ is assumed to be bounded, we will only consider the case of bounded operators $G_{0}, G_{1}$; this simplifies the exposition. We shall also assume $\mathcal{H}=\mathcal{K}$.

Let $H$ be a self-adjoint operator in a Hilbert space $\mathcal{H}$ and let $G$ be a bounded operator in $\mathcal{H}$. One says that $G$ is Kato smooth with respect to $H$ (we will write $G \in \operatorname{Smooth}(H))$, if

$$
\|G\|_{\operatorname{Smooth}(H)}:=\sup _{\|\varphi\|_{L^{2}(\mathbb{R})}=1}\|G \varphi(H)\|<\infty .
$$

As shown in [4], this definition coincides with the standard definition (see [6]) of Kato smoothness. The advantage of the definition (2.5) is that it extends naturally to Schatten classes. Generalising (2.5), we will say that $G \in \operatorname{Smooth}_{p}(H)$ for some $0<p<\infty$, if

$$
\|G\|_{\operatorname{Smooth}_{p}(H)}:=\sup _{\|\varphi\|_{L^{2}(\mathbb{R})}=1}\|G \varphi(H)\|_{p}<\infty .
$$

Finally, we shall write $G \in \operatorname{Smooth}_{\infty}(H)$, if $G \in \operatorname{Smooth}(H)$ and if

$$
G \mathbb{1}_{(-R, R)}(H) \in \mathbf{S}_{\infty} \quad \forall R>0 .
$$

It is very easy to prove [4, Lemma 2.3] that for $G \in \operatorname{Smooth}_{\infty}(H)$, one has

$$
G \varphi(H) \in \mathbf{S}_{\infty}, \quad \forall \varphi \in L^{2}(\mathbb{R}) .
$$


2.5. Main results from [4]. In the following theorem, $H_{0}$ and $H_{1}$ are self-adjoint operators in a Hilbert space $\mathcal{H}$ such that the perturbation $H_{1}-H_{0}$ factorises as

$$
H_{1}-H_{0}=G_{1}^{*} G_{0}
$$

where $G_{0}, G_{1}$ are bounded operators in $\mathcal{H}$. Let $\Lambda \subset \mathbb{R}$ be a measurable set; the case $\Lambda=\mathbb{R}$ is not excluded. (In fact, during the first reading of this subsection, the reader is encouraged to think of the simplest case $\Lambda=\mathbb{R}$.) Here we are interested in the "diagonal term" in (1.6),

$$
D_{\Lambda}(f):=\mathbb{1}_{\Lambda}\left(H_{1}\right) D(f) \mathbb{1}_{\Lambda}\left(H_{0}\right) .
$$

Since functions $f \in \operatorname{BMO}(\mathbb{R})$ in general need not be bounded, we need to take some care in defining the operator $D_{\Lambda}(f)$. We define the corresponding quadratic form

$$
d_{\Lambda, f}[u, v]:=\left(\mathbb{1}_{\Lambda}\left(H_{0}\right) u, \bar{f}\left(H_{1}\right) \mathbb{1}_{\Lambda}\left(H_{1}\right) v\right)-\left(f\left(H_{0}\right) \mathbb{1}_{\Lambda}\left(H_{0}\right) u, \mathbb{1}_{\Lambda}\left(H_{1}\right) v\right),
$$

for $u \in \operatorname{Dom} f\left(H_{0}\right), v \in \operatorname{Dom} f\left(H_{1}\right)$. Of course, if $f$ is bounded, we can define $D_{\Lambda}(f)$ directly and then

$$
d_{\Lambda, f}[u, v]=\left(D_{\Lambda}(f) u, v\right)
$$

for all $u$ and $v$ as above. We use the standard convention that if the norms in the right hand side of an upper bound are all finite, then the bound includes the statement that the norms in the left hand side are also finite. The following theorem is a combination of Theorems 7.5 and 7.6 from [4].

Theorem 2.1. Let $H_{0}, H_{1}, G_{0}, G_{1}, \Lambda, d_{\Lambda, f}$ be as above.

(i) For any $f \in \mathrm{BMO}(\mathbb{R})$, the sesquilinear form $d_{\Lambda, f}[u, v]$ satisfies the bound

$$
\left|d_{f}[u, v]\right| \leq C\|f\|_{\mathrm{BMO}(\mathbb{R})}\left\|G_{0} \mathbb{1}_{\Lambda}\left(H_{0}\right)\right\|_{\operatorname{Smooth}\left(H_{0}\right)}\left\|G_{1} \mathbb{1}_{\Lambda}\left(H_{1}\right)\right\|_{\operatorname{Smooth}\left(H_{1}\right)}\|u\|_{\mathcal{H}}\|v\|_{\mathcal{H}},
$$

for any $u \in \operatorname{Dom} f\left(H_{0}\right), v \in \operatorname{Dom} f\left(H_{1}\right)$, where the constant $C$ depends only on the choice of the norm in $\mathrm{BMO}(\mathbb{R})$. Thus, the form $d_{\Lambda, f}$ corresponds to a bounded linear operator $D_{\Lambda}(f)$ in $\mathcal{H}$ (in the sense of (2.7)), and this operator satisfies

$$
\left\|D_{\Lambda}(f)\right\| \leq C\|f\|_{\mathrm{BMO}(\mathbb{R})}\left\|G_{0} \mathbb{1}_{\Lambda}\left(H_{0}\right)\right\|_{\operatorname{Smooth}\left(H_{0}\right)}\left\|G_{1} \mathbb{1}_{\Lambda}\left(H_{1}\right)\right\|_{\operatorname{Smooth}\left(H_{1}\right)} .
$$

(ii) Assume that $G_{0} \mathbb{1}_{\Lambda}\left(H_{0}\right) \in \operatorname{Smooth}\left(H_{0}\right), G_{1} \mathbb{1}_{\Lambda}\left(H_{1}\right) \in \operatorname{Smooth}\left(H_{1}\right)$ and at least one of the inclusions

$$
G_{0} \mathbb{1}_{\Lambda}\left(H_{0}\right) \in \operatorname{Smooth}_{\infty}\left(H_{0}\right), \quad G_{1} \mathbb{1}_{\Lambda}\left(H_{1}\right) \in \operatorname{Smooth}_{\infty}\left(H_{1}\right)
$$

holds. Then for any $f \in \mathrm{CMO}(\mathbb{R})$ the operator $D_{\Lambda}(f)$ is compact.

(iii) Let $p, q, r$ be finite positive indices such that $\frac{1}{p}=\frac{1}{q}+\frac{1}{r}$. Then for any $f \in$ $B_{p, p}^{1 / p}(\mathbb{R}) \cap \operatorname{BMO}(\mathbb{R})$, one has

$\left\|D_{\Lambda}(f)\right\|_{p} \leq C(p)\|f\|_{B_{p, p}^{1 / p}(\mathbb{R})}\left\|G_{0} \mathbb{1}_{\Lambda}\left(H_{0}\right)\right\|_{\operatorname{Smooth}_{q}\left(H_{0}\right)}\left\|G_{1} \mathbb{1}_{\Lambda}\left(H_{1}\right)\right\|_{\operatorname{Smooth}_{r}\left(H_{1}\right)}$,

where the constant $C(p)$ depends only on the choice of the function $w$ in (2.2). 


\section{OfF-Diagonal terms}

Let $H_{0}, H_{1}$ be self-adjoint operators in $\mathcal{H}$, with

$$
H_{1}-H_{0}=G_{1}^{*} G_{0},
$$

where $G_{0}$ and $G_{1}$ are bounded operators in $\mathcal{H}$.

Let $\Lambda=(a-b, a+b)$ be a bounded open interval, and let $f \in \operatorname{BMO}(\mathbb{R})$ be a function supported in $\Lambda$. In this section we estimate the norms of the off-diagonal terms in (1.6),

$$
\mathbb{1}_{\Lambda^{c}}\left(H_{1}\right) f\left(H_{0}\right) \quad \text { and } \quad f\left(H_{1}\right) \mathbb{1}_{\Lambda^{c}}\left(H_{0}\right) .
$$

We set $\delta=\operatorname{dist}\left(\operatorname{supp} f, \Lambda^{c}\right)$ and denote $R_{0}(z)=\left(H_{0}-z\right)^{-1}, R_{1}(z)=\left(H_{1}-z\right)^{-1}$.

The following preliminary lemma establishes a series representation for the offdiagonal terms (3.1). This representation plays the same role here as the double operator integrals in the proof of Theorem 2.1 (see [4]): it allows us to estimate the operator norms of these terms. Then we will refine this representation and estimate the Schatten norms in Lemma 3.2 .

Lemma 3.1. Let $H_{0}, H_{1}, G_{0}, G_{1}, f, \Lambda$ be as described above. Assume that

$$
G_{0} \mathbb{1}_{\Lambda}\left(H_{0}\right) \in \operatorname{Smooth}\left(H_{0}\right) \quad \text { and } \quad G_{1} \mathbb{1}_{\Lambda}\left(H_{1}\right) \in \operatorname{Smooth}\left(H_{1}\right) \text {. }
$$

Then

$$
\begin{aligned}
& f\left(H_{1}\right) \mathbb{1}_{\Lambda^{c}}\left(H_{0}\right)=-\sum_{m=0}^{\infty} f\left(H_{1}\right)\left(H_{1}-a\right)^{m} G_{1}^{*} G_{0}\left(H_{0}-a\right)^{-m-1} \mathbb{1}_{\Lambda^{c}}\left(H_{0}\right), \\
& \mathbb{1}_{\Lambda^{c}}\left(H_{1}\right) f\left(H_{0}\right)=\sum_{m=0}^{\infty} \mathbb{1}_{\Lambda^{c}}\left(H_{1}\right)\left(H_{1}-a\right)^{-m-1} G_{1}^{*} G_{0}\left(H_{0}-a\right)^{m} f\left(H_{0}\right),
\end{aligned}
$$

where both series converge absolutely in the operator norm. For $z=a+i b$ we have the estimates

$$
\begin{aligned}
\left\|f\left(H_{1}\right) \mathbb{1}_{\Lambda^{c}}\left(H_{0}\right)\right\| & \leq \sqrt{2}(b / \delta)\|f\|_{L^{2}}\left\|G_{1} \mathbb{1}_{\Lambda}\left(H_{1}\right)\right\|_{\operatorname{Smooth}\left(H_{1}\right)}\left\|G_{0} R_{0}(z)\right\|, \\
\left\|\mathbb{1}_{\Lambda^{c}}\left(H_{1}\right) f\left(H_{0}\right)\right\| & \leq \sqrt{2}(b / \delta)\|f\|_{L^{2}}\left\|G_{0} \mathbb{1}_{\Lambda}\left(H_{0}\right)\right\|_{\operatorname{Smooth}\left(H_{0}\right)}\left\|G_{1} R_{1}(z)\right\| .
\end{aligned}
$$

Furthermore, if in addition

$$
G_{0} \mathbb{1}_{\Lambda}\left(H_{0}\right) \in \operatorname{Smooth}_{\infty}\left(H_{0}\right) \quad \text { and } \quad G_{1} \mathbb{1}_{\Lambda}\left(H_{1}\right) \in \operatorname{Smooth}_{\infty}\left(H_{1}\right) \text {, }
$$

then

$$
f\left(H_{1}\right) \mathbb{1}_{\Lambda^{c}}\left(H_{0}\right) \in \mathbf{S}_{\infty} \quad \text { and } \quad \mathbb{1}_{\Lambda^{c}}\left(H_{1}\right) f\left(H_{0}\right) \in \mathbf{S}_{\infty} .
$$


Proof. For simplicity of notation, we assume $a=0$ and let supp $f \subset\left[-b_{0}, b_{0}\right]$, $b_{0}=b-\delta$. First observe that formally, we have

$$
\begin{aligned}
\sum_{m=0}^{\infty} H_{1}^{m} G_{1}^{*} G_{0} H_{0}^{-m-1}=\sum_{m=0}^{\infty} H_{1}^{m}\left(H_{1}-H_{0}\right) H_{0}^{-m-1} & \\
& =\sum_{m=0}^{\infty}\left(H_{1}^{m+1} H_{0}^{-m-1}-H_{1}^{m} H_{0}^{-m}\right)=-I .
\end{aligned}
$$

After multiplication by $f\left(H_{1}\right)$ on the left and $\mathbb{1}_{\Lambda^{c}}\left(H_{0}\right)$ on the right, we obtain (3.2). Now let us prove the norm convergence of the series in (3.2). For each term, we have the estimate

$$
\begin{aligned}
\left\|f\left(H_{1}\right) H_{1}^{m} G_{1}^{*} G_{0} H_{0}^{-m-1} \mathbb{1}_{\Lambda^{c}}\left(H_{0}\right)\right\| \leq\left\|f\left(H_{1}\right) H_{1}^{m} G_{1}^{*}\right\|\left\|G_{0} H_{0}^{-m-1} \mathbb{1}_{\Lambda^{c}}\left(H_{0}\right)\right\| \\
\leq b_{0}^{m}\left\|f\left(H_{1}\right) G_{1}^{*}\right\| b^{-m}\left\|G_{0} H_{0}^{-1} \mathbb{1}_{\Lambda^{c}}\left(H_{0}\right)\right\| \\
\leq\left(b_{0} / b\right)^{m}\|f\|_{L^{2}}\left\|G_{1} \mathbb{1}_{\Lambda}\left(H_{1}\right)\right\|_{\operatorname{Smooth}\left(H_{1}\right)}\left\|G_{0} H_{0}^{-1} \mathbb{1}_{\Lambda^{c}}\left(H_{0}\right)\right\| .
\end{aligned}
$$

Since $b_{0}<b$, we have the norm convergence of the series in (3.2), and

$$
\sum_{m=0}^{\infty} b_{0}^{m} b^{-m}=1 /\left(1-b_{0} / b\right)=b / \delta
$$

gives the factor $b / \delta$ in (3.4). Finally,

$$
\left\|G_{0} H_{0}^{-1} \mathbb{1}_{\Lambda^{c}}\left(H_{0}\right)\right\| \leq\left\|G_{0} R_{0}(i b)\right\|\left\|H_{0}^{-1} \mathbb{1}_{\Lambda^{c}}\left(H_{0}\right)\left(H_{0}-i b\right)\right\| \leq \sqrt{2}\left\|G_{0} R_{0}(i b)\right\|,
$$

since

$$
\sup _{|\lambda|>b}|(\lambda-i b) / \lambda| \leq \sqrt{2}
$$

This gives the estimate (3.4).

The identity (3.3) and the estimate (3.5) are considered similarly. Finally, the compactness statement follows from the fact that by (2.6), each term in the norm convergent series (3.2), (3.3) is compact.

Now we come to the Schatten class estimate. It is not difficult to estimate the Schatten norm of the off-diagonal terms (3.1) by the expressions similar to the right sides of (3.4), (3.5) but with Schatten norms instead of the operator norms. However, in application to the Schrödinger operator, this is not sufficient, as the operators $G_{1} R_{1}(z), G_{0} R_{0}(z)$ will not necessarily be in the required Schatten classes. The standard way to deal with this problem is to consider the powers of the resolvent, i.e. to consider $G_{1} R_{1}(z)^{m}, G_{0} R_{0}(z)^{m}$ for sufficiently high $m$; these operators will be in the required Schatten class. This is what we do below. The price to pay are the additional terms in the r.h.s. of (3.8) and (3.9). 
Lemma 3.2. Assume the hypothesis of Lemma 3.1, and let $p, q, r$ be positive finite exponents satisfying $\frac{1}{p}=\frac{1}{q}+\frac{1}{r}$. Then for $z=a+i b$ and any integer $k \geq 0$,

$$
\begin{gathered}
\left\|f\left(H_{1}\right) \mathbb{1}_{\Lambda^{c}}\left(H_{0}\right)\right\|_{p} \leq C(b, \delta, p, k)\left(\|f\|_{L^{2}}\left\|G_{1} \mathbb{1}_{\Lambda}\left(H_{1}\right)\right\|_{\text {Smooth }_{r}\left(H_{1}\right)}\left\|G_{0} R_{0}(z)^{k+1}\right\|_{q}\right. \\
\left.+\left\|f\left(H_{1}\right)\left(R_{1}(z)^{k}-R_{0}(z)^{k}\right)\right\|_{p}\right), \\
\left\|\mathbb{1}_{\Lambda^{c}}\left(H_{1}\right) f\left(H_{0}\right)\right\|_{p} \leq C(b, \delta, p, k)\left(\|f\|_{L^{2}}\left\|G_{0} \mathbb{1}_{\Lambda}\left(H_{0}\right)\right\|_{\operatorname{Smooth}_{q}\left(H_{0}\right)}\left\|G_{1} R_{1}(z)^{1+k}\right\|_{r}\right. \\
\left.+\left\|\left(R_{0}(z)^{k}-R_{1}(z)^{k}\right) f\left(H_{0}\right)\right\|_{p}\right) .
\end{gathered}
$$

Proof. For simplicity of notation, we assume $a=0$ and let $\operatorname{supp} f \subset\left[-b_{0}, b_{0}\right]$, $b_{0}=b-\delta$. We will prove the first bound (3.8); the second bound (3.9) is proved in the same way.

Step 1. We prove the lemma for $k=0$.

We need to estimate the $\mathbf{S}_{p}$ norm of each term in the series in (3.2). Similarly to (3.6), we have

$$
\begin{array}{r}
\left\|f\left(H_{1}\right) H_{1}^{m} G_{1}^{*} G_{0} H_{0}^{-m-1} \mathbb{1}_{\Lambda^{c}}\left(H_{0}\right)\right\|_{p} \leq\left\|f\left(H_{1}\right) H_{1}^{m} G_{1}^{*}\right\|_{r}\left\|G_{0} H_{0}^{-m-1} \mathbb{1}_{\Lambda^{c}}\left(H_{0}\right)\right\|_{q} \\
\leq b_{0}^{m}\left\|f\left(H_{1}\right) G_{1}^{*}\right\|_{r} b^{-m}\left\|G_{0} H_{0}^{-1} \mathbb{1}_{\Lambda^{c}}\left(H_{0}\right)\right\|_{q} \\
\leq\left(b_{0} / b\right)^{m}\|f\|_{L^{2}}\left\|G_{1} \mathbb{1}_{\Lambda}\left(H_{1}\right)\right\|_{\operatorname{Smooth}_{r}\left(H_{1}\right)}\left\|G_{0} R_{0}(i b)\right\|_{q}\left\|\left(H_{0}-i b\right) H_{0}^{-1} \mathbb{1}_{\Lambda^{c}}\left(H_{0}\right)\right\| \\
\leq \sqrt{2}\left(b_{0} / b\right)^{m}\|f\|_{L^{2}}\left\|G_{1} \mathbb{1}_{\Lambda}\left(H_{1}\right)\right\|_{\operatorname{Smooth}_{r}\left(H_{1}\right)}\left\|G_{0} R_{0}(i b)\right\|_{q},
\end{array}
$$

where the last estimate uses (3.7). For $p \geq 1$, this yields

$$
\begin{array}{r}
\left\|f\left(H_{1}\right) \mathbb{1}_{\Lambda^{c}}\left(H_{0}\right)\right\|_{p} \leq \sum_{m=0}^{\infty} \sqrt{2}\left(b_{0} / b\right)^{m}\|f\|_{L^{2}}\left\|G_{1} \mathbb{1}_{\Lambda}\left(H_{1}\right)\right\|_{\operatorname{Smooth}_{r}\left(H_{1}\right)}\left\|G_{0} R_{0}(i b)\right\|_{q} \\
=\sqrt{2}(b / \delta)\|f\|_{L^{2}}\left\|G_{1} \mathbb{1}_{\Lambda}\left(H_{1}\right)\right\|_{\operatorname{Smooth}_{r}\left(H_{1}\right)}\left\|G_{0} R_{0}(i b)\right\|_{q} .
\end{array}
$$

For $0<p<1$ we use the modified triangle inequality (2.3) in $\mathbf{S}_{p}$, which yields the same estimate with a different constant. Thus we get the required estimate for $k=0$.

Step 2. We now consider $k>0$. Let $g(\lambda)=(\lambda-z)^{k} f(\lambda)$, so that

$$
f\left(H_{1}\right)=g\left(H_{1}\right) R_{0}(z)^{k}+g\left(H_{1}\right)\left(R_{1}(z)^{k}-R_{0}(z)^{k}\right)
$$

and therefore

$$
f\left(H_{1}\right) \mathbb{1}_{\Lambda^{c}}\left(H_{0}\right)=g\left(H_{1}\right) \mathbb{1}_{\Lambda^{c}}\left(H_{0}\right) R_{0}(z)^{k}+g\left(H_{1}\right)\left(R_{1}(z)^{k}-R_{0}(z)^{k}\right) \mathbb{1}_{\Lambda^{c}}\left(H_{0}\right) .
$$

Let us discuss the two terms on the right side of (3.10) separately.

The first term can be estimated by the same technique as in Step 1. This yields

$$
\begin{aligned}
\left\|g\left(H_{1}\right) \mathbb{1}_{\Lambda^{c}}\left(H_{0}\right) R_{0}(z)^{k}\right\|_{p} \leq C(b, \delta, p)\left\|G_{1} \bar{g}\left(H_{1}\right)\right\|_{r}\left\|G_{0} H_{0}^{-1} R_{0}(z)^{k} \mathbb{1}_{\Lambda^{c}}\left(H_{0}\right)\right\|_{q} \\
\leq C(b, \delta, p, k)\|f\|_{L^{2}}\left\|G_{1} \mathbb{1}_{\Lambda}\left(H_{1}\right)\right\|_{\text {Smooth }_{r}\left(H_{1}\right)}\left\|G_{0} R_{0}(z)^{k+1}\right\|_{q} .
\end{aligned}
$$


The second term in (3.10) is simply estimated by

$$
\left\|g\left(H_{1}\right)\left(R_{1}(z)^{k}-R_{0}(z)^{k}\right) \mathbb{1}_{\Lambda^{c}}\left(H_{0}\right)\right\|_{p} \leq 2^{k / 2} b^{k}\left\|f\left(H_{1}\right)\left(R_{1}(z)^{k}-R_{0}(z)^{k}\right)\right\|_{p} .
$$

This completes the proof of the lemma.

\section{4. $\mathbf{S}_{p}$ VAlued SMOOTHNESS FOR THE SCHRÖDINGER OPERATOR}

In this section $H_{0}, H_{1}$ are as in (1.1). We set $\langle x\rangle=\sqrt{1+|x|^{2}}$ and assume that $V(x)$ is real valued and satisfies the condition

$$
|V(x)| \leq C\langle x\rangle^{-\rho}, \quad \rho>1 .
$$

We denote the resolvents by $R_{0}(z)=\left(H_{0}-z\right)^{-1}, R_{1}(z)=\left(H_{1}-z\right)^{-1}$.

4.1. The LAP and its consequences. First we recall the limiting absorption principle (LAP) for the Schrödinger operator and translate it into statements about $\mathbf{S}_{p}$-valued smoothness.

Lemma 4.1. Let $H_{0}, H_{1}$ be as above, with some $\rho>1$. Then for any $\lambda>0$, the limits

$$
\langle x\rangle^{-\rho / 2} R_{0}(\lambda \pm i 0)\langle x\rangle^{-\rho / 2}, \quad\langle x\rangle^{-\rho / 2} R_{1}(\lambda \pm i 0)\langle x\rangle^{-\rho / 2}
$$

exist in the operator norm and are continuous (in the operator norm) in $\lambda>0$. Further, for any $p \geq 1, p>\frac{d-1}{\rho-1}$, we have the inclusions

$$
\begin{aligned}
& \operatorname{Im}\left(\langle x\rangle^{-\rho / 2} R_{0}(\lambda+i 0)\langle x\rangle^{-\rho / 2}\right) \in \mathbf{S}_{p}, \\
& \operatorname{Im}\left(\langle x\rangle^{-\rho / 2} R_{1}(\lambda+i 0)\langle x\rangle^{-\rho / 2}\right) \in \mathbf{S}_{p},
\end{aligned}
$$

and these operators are continuous in $\lambda>0$ in $\mathbf{S}_{p}$. Finally, for the same range of $p$ we have the inclusions

$$
\langle x\rangle^{-\rho / 2} \mathbb{1}_{\Lambda}\left(H_{0}\right) \in \operatorname{Smooth}_{2 p}\left(H_{0}\right), \quad\langle x\rangle^{-\rho / 2} \mathbb{1}_{\Lambda}\left(H_{1}\right) \in \operatorname{Smooth}_{2 p}\left(H_{1}\right)
$$

for any bounded interval $\Lambda \subset \mathbb{R}$ with $\operatorname{clos}(\Lambda) \subset(0, \infty)$.

Proof. The existence and continuity of the limits (4.2) is the standard LAP, see e.g. [14, Proposition 1.7.1, Theorem 6.2.1]. The inclusion (4.3) and the corresponding continuity in $\lambda>0$ is also well-known; see e.g. [14, Lemma 8.1.2].

In order to deal with the operator in (4.4), we need a version of the resolvent identity. For $\operatorname{Im} z>0$, we have

$$
R_{1}(z)=\left(I+R_{0}(z) V\right)^{-1} R_{0}(z), \quad\left(I+R_{0}(z) V\right)^{-1}=I-R_{1}(z) V .
$$

Taking the imaginary part in the first identity here and subsequently using the second identity, we obtain

$$
\begin{aligned}
\operatorname{Im} R_{1}(z)=\left(I+R_{0}(z) V\right)^{-1}(\operatorname{Im} & \left.R_{0}(z)\right)\left(I+V R_{0}(z)^{*}\right)^{-1} \\
& =\left(I-R_{1}(z) V\right)\left(\operatorname{Im} R_{0}(z)\right)\left(I-V R_{1}(z)^{*}\right) .
\end{aligned}
$$


Let us denote for brevity

$$
W(x)=\langle x\rangle^{-\rho / 2}, \quad V_{1}(x)=V(x)\langle x\rangle^{\rho / 2} .
$$

Multiplying (4.6) by $W$ both on the right and on the left, we obtain

$$
\begin{aligned}
\operatorname{Im}\left(W R_{1}(z) W\right)=W & \left(I-R_{1}(z) V\right)\left(\operatorname{Im} R_{0}(z)\right)\left(I-V R_{1}(z)^{*}\right) W \\
= & \left(I-W R_{1}(z) V_{1}\right) \operatorname{Im}\left(W R_{0}(z) W\right)\left(I-V_{1} R_{1}(z)^{*} W\right) .
\end{aligned}
$$

Now observe that $\left|V_{1}(x)\right| \leq C\langle x\rangle^{-\rho / 2}$, and so, by the LAP (4.2), we can pass to the limit in the operator norm on both sides of (4.7) as $z \rightarrow \lambda+i 0, \lambda>0$. By (4.2) and (4.3), this yields the inclusion (4.4) and the continuity in $\lambda>0$.

Let us prove the first inclusion in (4.5). By the LAP, for any $\varphi \in L^{2}(\mathbb{R}), \operatorname{supp} \varphi \subset$ $\Lambda$, we have

$$
W \varphi\left(H_{0}\right)\left(W \varphi\left(H_{0}\right)\right)^{*}=W\left|\varphi\left(H_{0}\right)\right|^{2} W=\frac{1}{\pi} \int_{\Lambda}|\varphi(\lambda)|^{2} \operatorname{Im}\left(W R_{0}(\lambda+i 0) W\right) d \lambda,
$$

and therefore, by (4.3),

$$
\left\|W \varphi\left(H_{0}\right)\right\|_{2 p}^{2}=\left\|W\left|\varphi\left(H_{0}\right)\right|^{2} W\right\|_{p} \leq \frac{1}{\pi} \sup _{\lambda \in \Lambda}\left\|\operatorname{Im} W R_{0}(\lambda+i 0) W\right\|_{p} \int_{\Lambda}|\varphi(\lambda)|^{2} d \lambda .
$$

This gives the inclusion $W \mathbb{1}_{\Lambda}\left(H_{0}\right) \in \operatorname{Smooth}_{2 p}\left(H_{0}\right)$. The second inclusion in (4.5) follows from (4.4) in the same way.

4.2. Estimates for $g(x) h(-i \nabla)$ and their consequences. Let us we recall two estimates for operators of the form

$$
g(x) h(-i \nabla) \quad \text { in } L^{2}\left(\mathbb{R}^{d}\right),
$$

where $g, h$ are complex-valued functions on $\mathbb{R}^{d}$ of the class to be specified below. Notation (4.8) is a common shorthand for operators defined by

$$
\varphi \mapsto g(x)(\widetilde{h \widehat{\varphi}})(x), \quad x \in \mathbb{R}^{d}, \quad \varphi \in L^{2}\left(\mathbb{R}^{d}\right),
$$

where $\varphi \mapsto \widehat{\varphi}$ is the standard (unitary) Fourier transform and $\varphi \mapsto \breve{\varphi}$ is the inverse Fourier transform. See e.g. [12, Chapter 4] for the details. For $q>0$ and a complex valued function $g$ on $\mathbb{R}^{d}$, we will use the notation

$$
\|g\|_{\ell^{q}\left(L^{2}\right)}^{q}:=\sum_{k \in \mathbb{Z}^{d}}\left(\int_{(0,1)^{d}+k}|g(x)|^{2} d x\right)^{q / 2} ;
$$

the space $\ell^{q}\left(L^{2}\right)$ is the set of functions $g$ with $\|g\|_{\ell^{q}\left(L^{2}\right)}<\infty$.

Proposition 4.2. (i) Let $2 \leq q<\infty$ and $g, h \in L^{q}\left(\mathbb{R}^{d}\right)$. Then $g(x) h(-i \nabla) \in \mathbf{S}_{q}$ and

$$
\|g(x) h(-i \nabla)\|_{q} \leq C_{d, q}\|g\|_{L^{q}}\|h\|_{L^{q}} .
$$

(ii) Let $0<q \leq 2$ and $g, h \in \ell^{q}\left(L^{2}\right)$. Then $g(x) h(-i \nabla) \in \mathbf{S}_{q}$ and

$$
\|g(x) h(-i \nabla)\|_{q} \leq C_{d, q}\|g\|_{\ell^{q}\left(L^{2}\right)}\|h\|_{\ell^{q}\left(L^{2}\right)} .
$$


Part (i) is the Kato-Seiler-Simon inequality, see [11] or [12, Thm. 4.1]; part (ii) is the Birman-Solomyak inequality, see [2, Thm. 11.1] (or [12, Thm. 4.5] for $1 \leq q \leq 2$ ). Part (ii) is used in the next lemma, and part (i) is used in the following Section.

Lemma 4.3. Let $\sigma>0$ and $d / \sigma<q \leq 2$. Then $\langle x\rangle^{-\sigma} \mathbb{1}_{\Lambda}\left(H_{0}\right) \in \operatorname{Smooth}_{q}\left(H_{0}\right)$ for any bounded interval $\Lambda \subset \mathbb{R}$ with $\operatorname{clos}(\Lambda) \subset(0, \infty)$.

Proof. Follows from Proposition 4.2(ii):

$$
\left\|\langle x\rangle^{-\sigma} \mathbb{1}_{\Lambda}\left(H_{0}\right) \varphi\left(H_{0}\right)\right\|_{q} \leq C\left\|\langle x\rangle^{-\sigma}\right\|_{\ell^{q}\left(L^{2}\right)}\left\|\varphi\left(|\xi|^{2}\right)\right\|_{\ell^{q}\left(L^{2}\right)} \leq C\|\varphi\|_{L^{2}}
$$

where $\operatorname{supp} \varphi \subset \operatorname{clos} \Lambda$.

\section{Global $\mathbf{S}_{p}$ COnditions}

Here $H_{0}, H_{1}, V$ are as in the previous section.

Lemma 5.1. Let $\sigma>0, q>0, m \in \mathbb{N}$ be such that

$$
\sigma q>d \quad \text { and } \quad 2 m q>d .
$$

Then for $\operatorname{Im} z \neq 0$, we have the inclusion $\langle x\rangle^{-\sigma} R_{0}(z)^{m} \in \mathbf{S}_{q}$. Further, if $f \in$ $\mathrm{BMO}(\mathbb{R})$ has compact support in $(0, \infty)$, then also $\langle x\rangle^{-\sigma} R_{0}(z)^{m} f\left(H_{0}\right) \in \mathbf{S}_{q}$.

Proof. For $q \geq 2$ we use Proposition 4.2(i):

$$
\left\|\langle x\rangle^{-\sigma} R_{0}(z)^{m}\right\|_{q}^{q} \leq C_{q, d}\left\|\langle x\rangle^{-\sigma}\right\|_{L^{q}}^{q}\left\|\left(|\xi|^{2}-z\right)^{-m}\right\|_{L^{q}}^{q} .
$$

This proves the first assertion since $\left\|\langle x\rangle^{-\sigma}\right\|_{L^{q}}<\infty$ if $\sigma q>d$ and $\left\|\left(|\xi|^{2}-z\right)^{-m}\right\|_{L^{q}}<$ $\infty$ if $2 m q>d$.

For $0<q<2$ we use Proposition 4.2 (ii):

$$
\left\|\langle x\rangle^{-\sigma} R_{0}(z)^{-m}\right\|_{q}^{q} \leq C_{d, q}\left\|\langle x\rangle^{-\sigma}\right\|_{\ell^{q}\left(L^{2}\right)}^{q}\left\|\left(|\xi|^{2}-z\right)^{-m}\right\|_{\ell^{q}\left(L^{2}\right)}^{q} .
$$

Again, we have $\left\|\langle x\rangle^{-\sigma}\right\|_{\ell^{q}\left(L^{2}\right)}<\infty$ if $\sigma q>d$ and $\left\|\left(|\xi|^{2}-z\right)^{-1}\right\|_{\ell^{q}\left(L^{2}\right)}<\infty$ if $2 m q>d$.

The assertion with an additional term in BMO follows in the same way since the $L^{q}$ or $\ell^{q}\left(L^{2}\right)$ norm of $\left(|\xi|^{2}-z\right)^{-1} f\left(|\xi|^{2}\right)$ is still finite if $2 m q>d$.

We also need an analogue of Lemma 5.1 with $R_{1}^{m}$ instead of $R_{0}^{m}$. In order to prove it, we need to consider the difference $R_{1}^{m}-R_{0}^{m}$. The following lemma is essentially contained in [13]. We include its proof for the sake of completeness.

Lemma 5.2. Let $V$ satisfy (4.1) with some $\rho>0$, let $r>0$ and let $m \geq 0$ be an integer such that

$$
\rho r>d \quad \text { and } \quad 2(m+1) r>d .
$$

Then for $\operatorname{Im} z \neq 0$ we have the inclusion $R_{1}(z)^{m}-R_{0}(z)^{m} \in \mathbf{S}_{r}$, and, if $f \in$ $\mathrm{BMO}(\mathbb{R})$ has compact support, then also $f\left(H_{0}\right)\left(R_{1}(z)^{m}-R_{0}(z)^{m}\right) \in \mathbf{S}_{r}$. 
Proof. Throughout the proof, we suppress the dependence on $z$, writing $R_{0}=$ $R_{0}(z)$ and $R_{1}=R_{1}(z)$. We use induction on $m$. For $m=0$ the statement is trivial. Now let $m \geq 1$ and assume the claim has already been proved for all smaller values of $m$. We have

$$
\begin{aligned}
R_{1}^{m}-R_{0}^{m}=\sum_{l=1}^{m} R_{1}^{l-1}\left(R_{1}-R_{0}\right) R_{0}^{m-l}=-\sum_{l=1}^{m} R_{1}^{l} V R_{0}^{m-l+1} & \\
& =-\left(\sum_{l=1}^{m} R_{0}^{l} V R_{0}^{m-l+1}+\sum_{l=1}^{m}\left(R_{1}^{l}-R_{0}^{l}\right) V R_{0}^{m-l+1}\right) .
\end{aligned}
$$

Separating the $l=m$ term in the second sum on the right, combining it with the left hand side and inverting $I+V R_{0}$ (the inverse exists and is bounded since $\operatorname{Im} z \neq 0$ ) we obtain

$$
R_{1}^{m}-R_{0}^{m}=-\left(\sum_{l=1}^{m} R_{0}^{l} V R_{0}^{m-l+1}+\sum_{l=1}^{m-1}\left(R_{1}^{l}-R_{0}^{l}\right) V R_{0}^{m-l+1}\right)\left(I+V R_{0}\right)^{-1} .
$$

Let us consider the first sum in the right hand side here. Let us check the inclusions

$$
R_{0}^{l} V R_{0}^{m-l+1} \in \mathbf{S}_{r}
$$

for each $1 \leq l \leq m$. We write

$$
R_{0}^{l} V R_{0}^{m-l+1}=\left(R_{0}^{l}|V|^{\alpha} \operatorname{sign}(V)\right)\left(|V|^{\beta} R_{0}^{m-l+1}\right)
$$

with $\alpha=\frac{l}{m+1}, \beta=\frac{m-l+1}{m+1}$. Setting $r_{1}=r(m+1) / l$ and $r_{2}=r(m+1) /(m-l+1)$, and using Lemma 5.1, we obtain

$$
R_{0}^{l}|V|^{\alpha} \in \mathbf{S}_{r_{1}}, \quad|V|^{\beta} R_{0}^{m-l+1} \in \mathbf{S}_{r_{2}} .
$$

Now (5.2) follows by application of the Hölder inequality in trace ideals (2.4).

Next, we consider the second sum in (5.1). Let us show the inclusion

$$
\left(R_{1}^{l}-R_{0}^{l}\right) V R_{0}^{m-l+1} \in \mathbf{S}_{r(m+1) /(m+2)} \subset \mathbf{S}_{r}
$$

for each $1 \leq l \leq m-1$. Let $r_{1}=r(m+1) /(l+1)$ and $r_{2}=r(m+1) /(m-l+1)$. Then $r_{1} \geq r$ and therefore $\rho r_{1}>d$. Moreover,

$$
2(l+1) r_{1}=2(m+1) r>d .
$$

Therefore, by induction hypothesis, $R_{1}^{l}-R_{0}^{l} \in \mathbf{S}_{r_{1}}$. On the other hand, $r_{2} \geq r$ and therefore $\rho r_{2}>d$. Moreover,

$$
2(m-l+1) r_{2}=2(m+1) r>d .
$$

Therefore, by Lemma 5.1, $V R_{0}^{m-l+1} \in \mathbf{S}_{r_{2}}$. By Hölder's inequality in trace ideals, since $r_{1}^{-1}+r_{2}^{-1}=((m+2) /(m+1)) r^{-1}$, we obtain the inclusion (5.3). Thus, the right hand side in (5.1) is in $\mathbf{S}_{r}$; we have completed the induction argument and thereby proved the first claim of the lemma. 
The second claim is proven in the same way: one checks without difficulty that (5.2), (5.3) hold true (for the same reasons as above) with an extra $f\left(H_{0}\right)$ term on the left.

Lemma 5.3. Let $\sigma>0, q>0, m \in \mathbb{N}$ be such that

$$
\rho q>d, \quad \sigma q>d \quad \text { and } \quad 2 m q>d .
$$

Then for $\operatorname{Im} z \neq 0$, we have the inclusion $\langle x\rangle^{-\sigma} R_{1}(z)^{m} \in \mathbf{S}_{q}$.

Proof. We write

$$
\langle x\rangle^{-\sigma} R_{1}(z)^{m}=\langle x\rangle^{-\sigma} R_{0}(z)^{m}+\langle x\rangle^{-\sigma}\left(R_{1}(z)^{m}-R_{1}(z)^{m}\right) .
$$

According to Lemma 5.1, the first term is in $\mathbf{S}_{q}$. The second term is in $\mathbf{S}_{q}$ by Lemma 5.2 (with $r=q$ ).

\section{Putting it AlL together}

Proof of Theorem 1.1. Throughout the proof, we set

$$
V=G_{1}^{*} G_{0}, \quad G_{0}=|V|^{1 / 2}, \quad G_{1}=\operatorname{sign}(V)|V|^{1 / 2},
$$

and let $\Lambda \subset \mathbb{R}$ be a bounded open interval such that supp $f \subset \Lambda$ and the closure of $\Lambda$ is contained in $(0, \infty)$. We consider the three terms in the right hand side of the decomposition (1.6).

First consider the diagonal term

$$
\mathbb{1}_{\Lambda}\left(H_{1}\right) D(f) \mathbb{1}_{\Lambda}\left(H_{0}\right) .
$$

By Lemma 4.1, we have

$$
G_{0} \mathbb{1}_{\Lambda}\left(H_{0}\right) \in \operatorname{Smooth}_{\infty}\left(H_{0}\right) \quad \text { and } \quad G_{1} \mathbb{1}_{\Lambda}\left(H_{1}\right) \in \operatorname{Smooth}_{\infty}\left(H_{1}\right) .
$$

Now we can use Theorem 2.1 , which ensures that for $f \in \operatorname{BMO}(\mathbb{R})$ the product (6.2) is bounded, and for $f \in \mathrm{CMO}(\mathbb{R})$ it is compact.

Next, the off-diagonal terms

$$
\mathbb{1}_{\Lambda^{c}}\left(H_{1}\right) f\left(H_{0}\right), \quad f\left(H_{1}\right) \mathbb{1}_{\Lambda^{c}}\left(H_{0}\right)
$$

are compact by Lemma 3.1 .

Proof of Theorem 1.2. Again, we decompose $f\left(H_{1}\right)-f\left(H_{0}\right)$ as in (1.6) and treat the three terms separately. Instead of following the cases (i) and (ii) as in the statement of the theorem, it will be convenient to split the range of variables as follows: $p \geq 1$ and $0<p<1$.

Case $p \geq 1$. Throughout the consideration of this case we use the factorisation (6.1). Observe that for $p \geq 1$ both in case (i) and in case (ii) we have

$$
p>\frac{d}{\rho} \quad \text { and } \quad p>\frac{d-1}{\rho-1} .
$$


The diagonal term. We use Theorem 2.1(iii) and take $q=r=2 p$. Both terms $\left\|G_{0} \mathbb{1}_{\Lambda}\left(H_{0}\right)\right\|_{\operatorname{Smooth}_{2 p}\left(H_{0}\right)}$ and $\left\|G_{1} \mathbb{1}_{\Lambda}\left(H_{1}\right)\right\|_{\text {Smooth }_{2 p}\left(H_{1}\right)}$ are finite as shown in Lemma 4.1.

The term $\mathbb{1}_{\Lambda^{c}}\left(H_{1}\right) f\left(H_{0}\right)$. Let $k \geq 0$ be an integer sufficiently large such that $2(k+1) p>d$. We use the bound (3.9) from Lemma 3.2. As already mentioned, the norm $\left\|G_{0} \mathbb{1}_{\Lambda}\left(H_{0}\right)\right\|_{\operatorname{Smooth}_{2 p}\left(H_{0}\right)}$ is finite. Moreover, according to Lemma 5.3, the assumptions $\rho p>d$ and $4(k+1) p>d$ imply that $G_{1} R_{1}(z)^{k+1} \in \mathbf{S}_{2 p}$ for $\operatorname{Im} z \neq 0$. If $k=0$, this already shows that $\mathbb{1}_{\Lambda^{c}}\left(H_{1}\right) f\left(H_{0}\right) \in \mathbf{S}_{p}$.

If $k \geq 1$, we still need to show that $\left(R_{0}(z)^{k}-R_{1}(z)^{k}\right) f\left(H_{0}\right) \in \mathbf{S}_{p}$. This follows from Lemma 5.2 (by taking adjoints).

The term $f\left(H_{1}\right) \mathbb{1}_{\Lambda^{c}}\left(H_{0}\right)$. The argument in this case is similar to that for the second term and we will be brief. We choose $k$ as before and this time, we use bound (3.8) from Lemma 3.2. We already know that $G_{1} \mathbb{1}_{\Lambda}\left(H_{1}\right) \in \operatorname{Smooth}_{2 p}\left(H_{1}\right)$ and we infer that $G_{0} R_{0}(z)^{k+1} \in \mathrm{S}_{2 p}$ from Lemma 5.1. This concludes the proof for $k=0$.

For $k \geq 1$, we still need to show that $f\left(H_{1}\right)\left(R_{1}(z)^{k}-R_{0}(z)^{k}\right) \in \mathbf{S}_{p}$. We write

$$
f\left(H_{1}\right)\left(R_{1}(z)^{k}-R_{0}(z)^{k}\right)=f\left(H_{0}\right)\left(R_{1}(z)^{k}-R_{0}(z)^{k}\right)+D(f)\left(R_{1}(z)^{k}-R_{0}(z)^{k}\right) .
$$

Since $f$ is compactly supported and $f \in B_{p, p}^{1 / p}$, we have $f \in$ BMO and therefore the operator $D(f)$ is bounded by Theorem 1.1. Thus, it suffices to prove that

$$
f\left(H_{0}\right)\left(R_{1}(z)^{k}-R_{0}(z)^{k}\right), R_{1}(z)^{k}-R_{0}(z)^{k} \in \mathbf{S}_{p} .
$$

This is again a consequence of Lemma 5.2 .

Case $0<p<1$. Here we are in the setting of part (ii) where $\rho>d$. Again, we treat separately the three terms in (1.6). This time we split the perturbation $V=G_{1}^{*} G_{0}$ with

$$
G_{0}=(\operatorname{sgn} V)|V|^{\theta} \quad \text { and } \quad G_{1}=|V|^{1-\theta} .
$$

Here $0<\theta<1$ is chosen such that, with $q=2 p /(2-p)$, we have $\theta \rho q>d$ and $(1-\theta) \rho>d / 2$. (Such choice of $\theta$ is possible since $p>d / \rho$.)

The diagonal term. We use Theorem 2.1(iii) with $q=2 p /(2-p)$ and $r=2$. The term $\left\|G_{0} \mathbb{1}_{\Lambda}\left(H_{0}\right)\right\|_{\operatorname{Smooth}_{q}\left(H_{0}\right)}$ is finite by Lemma 4.3 since $\theta \rho q>d$. Let us check that the term $\left\|G_{1} \mathbb{1}_{\Lambda}\left(H_{1}\right)\right\|_{\text {Smooth }_{2}\left(H_{1}\right)}$ is finite.

Let $\widetilde{\rho}=\min \{\rho, 2(1-\theta) \rho\}$. Then $V$ satisfies (4.1) with $\widetilde{\rho}$ instead of $\rho$. Moreover, $\widetilde{\rho}>1$ (since $\rho>1$ and $2(1-\theta) \rho>d \geq 1$ ) and $1>(d-1) /(\widetilde{\rho}-1)$ (since $\rho>d$ and $2(1-\theta) \rho>d)$. Therefore, we can apply Lemma 4.1 with $p=1$ and with $\widetilde{\rho}$ instead of $\rho$. This gives $\langle x\rangle^{-\widetilde{\rho} / 2} \mathbb{1}_{\Lambda}\left(H_{1}\right) \in \operatorname{Smooth}_{2}\left(H_{1}\right)$. On the other hand, $|V|^{1-\theta}\langle x\rangle^{\tilde{\rho} / 2}$ is bounded and therefore $G_{1} \mathbb{1}_{\Lambda}\left(H_{1}\right) \in \operatorname{Smooth}_{2}\left(H_{1}\right)$.

The term $\mathbb{1}_{\Lambda^{c}}\left(H_{1}\right) f\left(H_{0}\right)$. Let $k \geq 0$ be an integer sufficiently large so that $2(k+1) p>d$. We use bound (3.9) with the exponents $q=2 p /(2-p), r=2$. We already know that $G_{0} \mathbb{1}_{\Lambda}\left(H_{0}\right) \in \operatorname{Smooth}_{q}\left(H_{0}\right)$. Further, according to Lemma 5.3 , 
the assumptions $(1-\theta) \rho>d / 2$ and $4(k+1)>d$ imply that $G_{1} R_{1}(z)^{k+1} \in \mathbf{S}_{2}$ for $\operatorname{Im} z \neq 0$. If $k=0$, this already shows that $\mathbb{1}_{\Lambda^{c}}\left(H_{1}\right) f\left(H_{0}\right) \in \mathbf{S}_{p}$.

If $k \geq 1$, we argue as in the case $p \geq 1$ that $\left(R_{0}(z)^{k}-R_{1}(z)^{k}\right) f\left(H_{0}\right) \in \mathbf{S}_{p}$.

The term $f\left(H_{1}\right) \mathbb{1}_{\Lambda^{c}}\left(H_{0}\right)$. Again, the argument is similar and we will be brief. We choose $k$ as before and this time, we use bound (3.8). We already know that $G_{1} \mathbb{1}_{\Lambda}\left(H_{1}\right) \in \operatorname{Smooth}_{2}\left(H_{1}\right)$, and we infer that $G_{0} R_{0}(z)^{k+1} \in \mathbf{S}_{q}$ from Lemma 5.1 since $\theta \rho q>d$ and $2(k+1) q>d$. If $k=0$, this already shows that $f\left(H_{1}\right) \mathbb{1}_{\Lambda^{c}}\left(H_{0}\right) \in$ $\mathbf{S}_{p}$.

If $k \geq 1$, we argue as in the case $p \geq 1$ that $f\left(H_{1}\right)\left(R_{1}(z)^{k}-R_{0}(z)^{k}\right) \in \mathbf{S}_{p}$. This concludes the proof of the theorem.

\section{REFERENCES}

[1] J.Bergh, J.LÖFström, Interpolation spaces, Springer 1976.

[2] M. Sh. Birman, M. Z. Solomyak, Estimates for the singular numbers of integral operators. (Russian) Uspekhi Mat. Nauk 32 (1977), no. 1, 17-84. English transl. in Russian Math. Surveys 32 (1977), no. 1, 15-89.

[3] R. Frank, A. Pushnitski, Trace class conditions for functions of Schrödinger operators, Comm. Math. Phys. 335 (2015), 477-496.

[4] R. Frank, A. Pushnitski, Kato smoothness and functions of perturbed self-adjoint operators, preprint, arXiv:1901.04731.

[5] D. Girela, Analytic functions of bounded mean oscillation, Complex function spaces (Mekrijärvi, 1999), pp. 61-170.

[6] T. Kato, Wave operators and similarity for some non-selfadjoint operators, Math. Ann. $162(1965 / 1966), 258-279$.

[7] V. V. PELLER, Hankel operators in the theory of perturbations of unitary and self-adjoint operators, Funct. Anal. Appl. 19 (1985) 111-123.

[8] D. Potapov, F. Sukochev, Operator-Lipschitz functions in Schatten-von Neumann classes, Acta Math. 207 (2011), no. 2, 375-389.

[9] A. Pushnitski, D. YAfaev, Best rational approximation of functions with logarithmic singularities, Constr. Approx. 46 (2017), 243-269.

[10] A. Pushnitski, D. Yafaev, Spectral theory of piecewise continuous functions of self-adjoint operators, Proc. Lond. Math. Soc. (3) 108, no. 5, 1079-1115 (2014).

[11] E. Seiler, B. Simon, Bounds in the Yukawa 2 quantum field theory: upper bound on the pressure, Hamiltonian bound and linear lower bound, Comm. Math. Phys. 45 (1975), no. 2, 99-114.

[12] B. Simon, Trace Ideals and Their Applications, American Mathematical Society, Providence, RI, 2005.

[13] D.YAFAEV, A remark concerning the theory of scattering for a perturbed polyharmonic operator. (Russian) Mat. Zametki 15 (1974), 445-454. English transl. in Math. Notes 15 (1974), 260-265.

[14] D.Yafaev, Mathematical Scattering Theory: Analytic Theory, American Mathematical Society, Providence, RI, 2010. 
(Rupert L. Frank) Mathematisches Institut, Ludwig-Maximilans Universität München, Theresienstr. 39, 80333 München, Germany, and Department of Mathematics, California Institute of Technology, Pasadena, CA 91125, USA

E-mail address: rlfrank@caltech.edu

(Alexander Pushnitski) Department of Mathematics, King's College London, Strand, London, WC2R 2LS, UK

E-mail address: alexander.pushnitski@kcl.ac.uk 